\title{
Test of the Schrödinger functional with chiral fermions in the Gross-Neveu model
}

\author{
Björn Leder $* \dagger$ \\ Humboldt Universität zu Berlin, Germany
}

$H U-E P-07 / 56$

SFB/CPP-07-72

E-mail: leder@maths.tcd.ie

The recently proposed construction of chiral fermions on lattices with boundaries is tested in an interacting theory up to first order of perturbation theory. We confirm that, in the bulk of the lattice, the chiral Ward identities take their continuum value up to cutoff effects without any tuning. Universal quantities are defined that have an expansion in the renormalised couplings with coefficients that are functions of the physical size and the periodicity in the spatial direction. These coefficient functions have to be identical for different discretisations. We find agreement with the standard Wilson fermions. The computation is done in the asymptotically free GrossNeveu model with continuous chiral symmetry.

The XXV International Symposium on Lattice Field Theory

July 30-4 August 2007

Regensburg, Germany

\footnotetext{
${ }^{*}$ This work was supported by Deutsche Forschungsgemeinschaft in form of Sonderforschungsbereich SFB TR 09.

$\dagger$ current address: Trinity College Dublin, Dublin 2, Ireland
} 


\section{Introduction}

In this talk I present a 1-loop perturbative calculation that confirms the favourable properties of chiral fermions on a lattice with boundaries [1]. The calculation is performed in the chiral GrossNeveu model [2]. Before I go into the details and results of the calculation let me briefly introduce field theories on lattices with boundaries.

In the Schrödinger functional (SF) of a quantum field theory the fields are defined on a $d+1$ dimensional cylinder. The fields are subject to Dirichlet boundary conditions in the time direction $\left(P_{ \pm}=\frac{1}{2}\left(1 \pm \gamma_{0}\right)\right)$

$$
P_{+} \psi(x)=0, \quad \bar{\psi}(x) P_{-}=0 \quad \text { at } \quad x_{0}=0 \quad \text { and } \quad P_{-} \psi(x)=0, \quad \bar{\psi}(x) P_{+}=0 \quad \text { at } \quad x_{0}=T
$$

and periodic boundary conditions in the space directions $\psi(x+\hat{L})=\mathrm{e}^{i \theta} \psi(x), \theta \in[0,2 \pi)$.

Discretising such a theory on a space-time lattice with boundaries has advantages. If the temporal extension is a multiple of the spatial extension, say $T=2 L$, the inverse of the spatial extension $1 / L$ provides an infrared cutoff and is the natural energy scale in the massless theory. In fact this has enabled a fully non-perturbative determination of the scale dependence of the fundamental parameters of $N_{\mathrm{f}}=2 \mathrm{QCD}$, see [3] and references therein.

However, naively one would expect that theories become more complicated on lattices with boundaries. First of all the distinction between different classes of boundary conditions, for example Dirichlet and Neuberger boundary conditions, really only makes sense for smooth fields, which we do not have on the lattice. The boundary conditions therefore must be encoded in the lattice action and will arise dynamically in the continuum limit. In general this might involve the necessity to fine-tune some parameter in the action. Secondly the boundaries might cause additional divergences and thus lead to additional counter-terms. Both of these issues have been addressed for QCD in [4] with the result that there is no need for fine-tuning and that one just has to add a renormalisation factor for the boundary fields.

The fact that the SF boundary conditions arise naturally (without fine-tuning) in the continuum limit has been understood on the basis of dimensional counting and boundary critical phenomena, see [1] and references therein. The SF boundary conditions are part of the definition of the continuum limit. Together with the symmetry properties and the dimensionality of the system they form a SF universality class: any local discretisation has this continuum limit. ${ }^{1}$

Exactly this observation led the author of [1] to a formulation of the lattice SF of QCD with chiral fermions. In the continuum the SF boundary conditions break chiral symmetry. Therefore lattice fermions that fulfil the Ginsparg-Wilson relation [6] on the whole lattice (with boundaries) can not have the right continuum limit. The minimal modification to the Ginsparg-Wilson relation that breaks the lattice chiral symmetry at the boundary would be a term $\Delta_{B}$ that is supported at the boundary with exponentially decaying tails

$$
\gamma_{5} D+D \gamma_{5}=a D \gamma_{5} D+\Delta_{B}
$$

Given a local solution to this equation chiral Ward identities are expected to take their continuum form far away from the boundaries.

\footnotetext{
${ }^{1}$ Note that in [5] a SF is proposed with chirally rotated boundary conditions, which however break parity and therefore are distinct from the boundary conditions (1.1) considered here.
} 
As mentioned above, in principle there are many possible local lattice formulations that have the same continuum limit. In [1] the author gives one solution to the modified Ginsparg-Wilson relation, a modified Neuberger-Dirac operator

$$
D=\frac{1}{\bar{a}}\left\{1-\frac{1}{2}\left(U+\gamma_{5} U^{\dagger} \gamma_{5}\right)\right\}, \quad U=A\left(A^{\dagger} A+c a P\right)^{-1 / 2}, \quad \bar{a}=\frac{a}{1+s}, \quad A=1+s-a D_{\mathrm{W}},
$$

where $D_{\mathrm{W}}$ is the massless Wilson-Dirac operator in the presence of the boundaries as introduced in [4], i.e. standard infinite lattice Wilson-Dirac operator in the range $0<x_{0}<T$ and at all other times the target field $\chi=D_{W} \psi$ is set to zero. ${ }^{2}$ The projector $P$ is defined through

$$
P \psi(x)=\frac{1}{a}\left\{\left.\delta_{x_{0}, a} P_{-} \psi(x)\right|_{x_{0}=a}+\left.\delta_{x_{0}, T-a} P_{+} \psi(x)\right|_{x_{0}=T-a}\right\},
$$

and the parameters $c$ and $s$ must be chosen such as to ensure locality (see next section). The operator (1.3) solves (1.2) if the lattice spacing $a$ is replaced by the rescaled value $\bar{a}$. For all unexplained notation we refer the reader to [7].

\section{Free fermions}

For Wilson fermions the free propagator in presence of the boundaries can be calculated explicitly and is given in [8]. In the case of chiral fermions we do not even have an analytic expression for the Dirac operator. To obtain the free propagator in closed form like in the Wilson case might be possible but is much more difficult. Nevertheless, the operator under the square root in (1.3) can be worked out in the time-momentum representation.

In the free theory the operator under the square root explicitly reads

$$
A^{\dagger} A+c a P=(1+s)^{2}+s a^{2} \sum_{\mu} \partial_{\mu}^{*} \partial_{\mu}+\frac{1}{2} a^{4} \sum_{\mu<v} \partial_{\mu}^{*} \partial_{\mu} \partial_{v}^{*} \partial_{v}+(c-1) a P,
$$

It is Hermitian and therefore has real eigenvalues. Due to translation invariance in space the spatial eigenfunctions are plane waves with momentum values that are integer multiples of $2 \pi / L$ in the range $-\pi / a<p_{k} \leq \pi / a$. The eigenfunctions of the operator (2.1) are then given by

$$
\begin{gathered}
w_{p_{0}, \mathbf{p}}(x)=\mathrm{e}^{i \mathbf{p x}}\left\{P_{-} \sin \left(p_{0} x_{0}+b\left(p_{0}\right)\right)+P_{+} \sin \left(p_{0}\left(T-x_{0}\right)+b\left(p_{0}\right)\right)\right\}, \\
b\left(p_{0}\right)=-\arctan \left(\frac{\sin \left(a p_{0}\right)}{\frac{q}{c-1}+\cos \left(a p_{0}\right)}\right) .
\end{gathered}
$$

The allowed values of $p_{0}$ are given by the solutions of the transcendental equation $b\left(p_{0}\right)=-p_{0} T$. The corresponding eigenvalues are given by

$$
\lambda_{p_{0}, \mathbf{p}}=(1+s)^{2}-s a^{2} \hat{p}^{2}+\frac{a^{4}}{2} \sum_{\mu<v} \hat{p}_{\mu}^{2} \hat{p}_{v}^{2}
$$

One can easily show that the eigenvalues are bounded from below by $(1-|s|)^{2}$ for $|s|<1$ and $c \geq 1$. Adopting the argument in ref. [9], using expansion in Legendre polynomials, we conclude that in the free theory the locality of the Dirac operator (1.3) guaranteed for this range of parameter values.

\footnotetext{
${ }^{2}$ This Dirac operator maps the space of fields defined at all $x_{0}$, but are zero at $x_{0}<a$ and $x_{0}>T-a$, into itself.
} 
The eigenfunctions (2.2) can be orthonormalised and used to write down an analytical expression for the kernel $D(x, y)$ of the Dirac operator. But the evaluation of $D(x, y)$ in this way would be very expensive, since it involves a sum over momenta $p_{0}$ which in turn are determined for each set of parameter values by the roots of a transcendental equation.

In our computation we used a different approach. First the operator (1.3) is half-Fouriertransformed to its time-momentum representation $D\left(x_{0}, \mathbf{p}\right)$. The square-root of the remaining $(d+1)(T-1) \times(d+1)(T-1)$-matrices (one for each value of $\mathbf{p})$ is computed with the minmax polynomial as explained in section 4 of [10]. For the final step, to obtain the propagator, we used the built-in inversion routine of MATLAB.

The so calculated modified Neuberger-Dirac operator has been inserted in the Ginsparg-Wilson relation to compute the difference $\Delta_{B}$ and to confirm, that it is in fact localised at the boundaries with exponentially decaying tails.

\section{Chiral Gross-Neveu model}

To test the operator (1.3) beyond free fermions we introduce a two-dimensional theory with four-fermion interactions. The euclidean continuum action of the chiral Gross-Neveu model can be given in the form

$$
\begin{gathered}
S_{\mathrm{CGN}}^{c}=\int \mathrm{d} x^{2}\left\{\bar{\psi} \gamma_{\mu} \partial_{\mu} \psi-\frac{1}{2} g^{2}\left(O_{S S}-O_{P P}\right)-\frac{1}{2} g_{V}^{2} O_{V V}\right\} \\
O_{S S}=(\bar{\psi} \psi)^{2}, \quad O_{P P}=\left(\bar{\psi} \gamma_{5} \psi\right)^{2}, \quad O_{V V}=\sum_{\mu}\left(\bar{\psi} \gamma_{\mu} \psi\right)^{2} .
\end{gathered}
$$

All Dirac and flavour indices are suppressed and contracted in a straightforward way. For $N$ flavours of fermions this action possesses an $U(N)$-flavour symmetry and an $U(1)$ continuous chiral symmetry. For a detailed derivation of the possible terms (in terms of renormalisability) see chapter 5 of ref. [7]. The terms in (3.1) are a full set of allowed terms that respect the above mentioned symmetries (plus euclidean symmetry). For $N \geq 2$ the model shares with QCD the property of an asymptotically free coupling (namely $g^{2}$ ).

Discretisation with Wilson fermions is straightforward. But since the Wilson term breaks chiral symmetry a mass term and an additional coupling have to be added

$$
S_{\mathrm{CGN}, \mathrm{W}}=a^{2} \sum_{x}\left\{\bar{\psi}\left(D_{\mathrm{W}}+m_{0}\right) \psi-\frac{1}{2} g_{w}^{2}\left(O_{S S}-O_{P P}\right)-\frac{1}{2} \delta_{P}^{2} O_{P P}-\frac{1}{2} g_{V, w}^{2} O_{V V}\right\} .
$$

No additional coupling (or mass term) is necessary if one uses a Dirac operator that solves the Ginsparg-Wilson relation. Since such an operator comes with a lattice chiral symmetry [11], there are no other allowed dimension 2 operators. However, to make the action manifestly invariant under that symmetry, one has to add irrelevant dimension 3 operators via the substitution $\psi \rightarrow \hat{\psi}=$ $\left(1-\frac{a}{2} D\right) \psi$ in the four-fermion interaction terms. The action then reads

$$
S_{\mathrm{CGN}, \mathrm{GW}}=a^{2} \sum_{x}\left\{\bar{\psi} D \psi-\frac{1}{2} g_{g w}^{2}\left(\hat{O}_{S S}-\hat{O}_{P P}\right)-\frac{1}{2} g_{V, g w}^{2} \hat{O}_{V V}\right\} .
$$

Defining now the SF of the Gross-Neveu model is straightforward. Since it is asymptotically free (for $N \geq 2$, which we assume from now on), the scaling dimension of local fields is equal to their engineering dimension. Therefore the argument about the naturalness of the SF boundary conditions in QCD [1] holds also in the chiral Gross-Neveu model. And in [7] it is shown that just one additional renormalisation factor for the boundary fields has to be added. 


\section{Chiral Ward identity}

The continuum chiral Ward identity

$$
\left\langle\partial_{\mu} A_{\mu}(x) \mathscr{O}(y)\right\rangle=2 m\langle P(x) \mathscr{O}(y)\rangle, \quad x \neq y
$$

implies on the lattice for vanishing renormalised mass $m_{R}=0$

$$
\left\langle(\mathscr{O})_{\mathrm{R}} \widetilde{\partial}_{\mu}\left(A_{\mu}\right)_{\mathrm{R}}(x)\right\rangle=\mathrm{O}(a)
$$

This can be used to compute the critical mass $m_{c}$ and the symmetric coupling $\delta_{P, s}$ in (3.3) to second order in perturbation theory (PT) [7].

As indicated in (4.2) there are chiral symmetry breaking effects that are lattice artifacts. In this section we compute these effects for Wilson fermions and the proposed chiral fermions. To this end correlation functions that match the operators in (4.1) and (4.2) have to be defined.

Correlation functions like

$$
f_{A}\left(x_{0}\right)=-\frac{a^{2}}{2 N} \sum_{y_{1}, z_{1}}\left\langle\bar{\psi}(x) \gamma_{0} \gamma_{5} \psi(x) \bar{\zeta}\left(y_{1}\right) \gamma_{5} \zeta\left(z_{1}\right)\right\rangle
$$

correlate boundary states (here a pseudo-scalar) with current insertions (here axial current). The boundary fields $\zeta\left(x_{1}\right)=P_{-} \psi(x)$ and $\bar{\zeta}\left(x_{1}\right)=\bar{\psi}(x) P_{+}$at $x_{0}=a$ become the non-vanishing components of $\psi, \bar{\psi}$ at boundary $x_{0}=0$ in the continuum limit. Similarly $f_{P}\left(x_{0}\right)$ is defined to correlate a pseudo-scalar boundary state to a pseudo-scalar density insertion.

With the help of these two correlation functions the bare current mass can be computed in PT

$$
r\left(x_{0}\right)=\frac{a \tilde{\partial}_{0} f_{A}\left(x_{0}\right)}{2 f_{P}\left(x_{0}\right)}=r_{0}\left(x_{0}\right)+r_{1}\left(x_{0}\right) g^{2}+\mathrm{O}\left(g^{4}\right)
$$

If the renormalised mass is set to zero $\left(m_{R}=0\right)$ this quantity is a direct measure of the lattice artifacts on the right hand side of (4.2).

At tree-level this quantity vanishes identically for Wilson and for chiral fermions. At 1-loop the behaviour is similar for Wilson and chiral fermions. In fact, for the proposed chiral fermions the chiral symmetry breaking effects are localised at the boundary and decay exponentially with the distance to the boundaries, see fig. 1 .

\section{Renormalised coupling}

Having confirmed the desired chiral properties of the proposed chiral fermions in the SF, we now renormalise the couplings at vanishing renormalised mass and define universal quantities that have a finite and unique continuum limit.

To this end we define boundary to boundary correlation functions

$$
\begin{gathered}
f_{4}=-\frac{a^{4}}{2\left(N^{2}-1\right) L^{2}} \sum_{u_{1} v_{1} y_{1} z_{1}}\left\langle\bar{\zeta}^{\prime}\left(u_{1}\right) \Gamma_{B}^{\prime} \lambda^{a} \zeta^{\prime}\left(v_{1}\right) \bar{\zeta}\left(y_{1}\right) \Gamma_{B} \lambda^{a} \zeta\left(z_{1}\right)\right\rangle \\
f_{2}=-\frac{a^{2}}{N L} \sum_{u_{1} z_{1}}\left\langle\bar{\zeta}\left(u_{1}\right) \zeta^{\prime}\left(z_{1}\right)\right\rangle, \quad\left(f_{2}\right)_{\mathrm{R}}=Z_{\zeta}^{2} f_{2} \quad \text { and } \quad\left(f_{4}\right)_{\mathrm{R}}=Z_{\zeta}^{4} f_{4}
\end{gathered}
$$



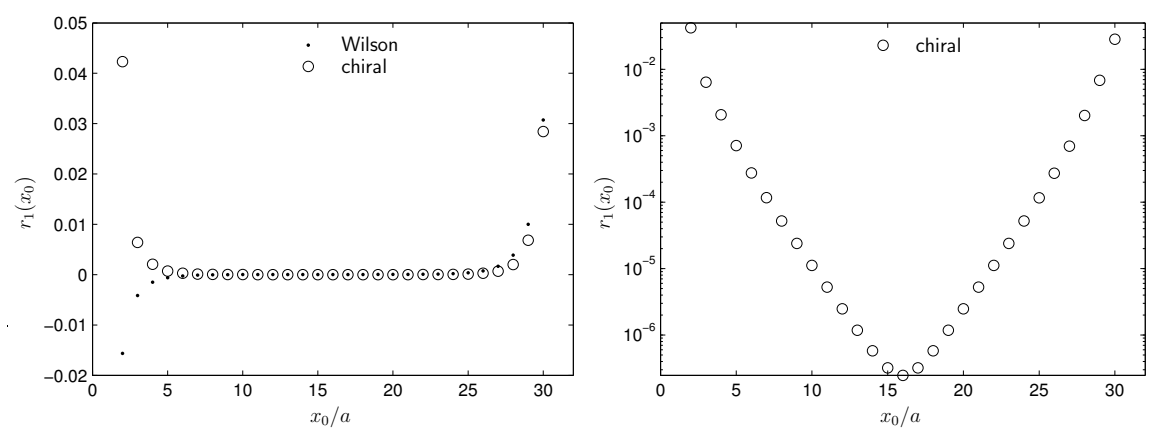

Figure 1: Bare current mass at 1-loop in PT (left). Logarithmic plot of this quantity for chiral fermions (right). The plots are for a $16 \times 32$ lattice, $\theta=0, N=2$ and $g_{V}^{2}=0$.

and the renormalisation factor free ratio

$$
R(\theta)=\frac{\left(f_{4}\right)_{\mathrm{R}}}{\left(f_{2}\right)_{\mathrm{R}}^{2}}-1=\frac{f_{4}}{\left(f_{2}\right)^{2}}-1 \quad \text { at } \quad m_{R}=0
$$

which only depends on the periodicity angle $\theta$ in the spatial directions. The $\lambda^{a} \mathrm{~s}$ are the generators of the algebra of $S U(N)$ and $\zeta^{\prime}, \bar{\zeta}^{\prime}$ are the equivalents of $\zeta, \bar{\zeta}$ at $x_{0}=T-a$.

Renormalised couplings can now be defined (up to normalisation factors) as the difference of $R(\theta)$ at two values of $\theta$

$$
\tilde{g}^{2} \propto R\left(\theta_{1}\right)-R\left(\theta_{2}\right)
$$

and similar for $\tilde{g}_{V}^{2}$ [7]. For definiteness we fix one of them to $\theta_{1}=0$. There are then renormalised couplings $\tilde{g}_{\eta}^{2}, \tilde{g}_{V, \eta}^{2}$ for each value $\theta_{2}=\eta$. They have an expansion in PT and the 1-loop result for $\tilde{g}_{\eta}^{2}$ is

$$
\tilde{g}_{\eta}^{2} \stackrel{a \rightarrow 0}{=} g_{w}^{2}+g_{w}^{4} b_{0} \ln (a / L)+c_{g}^{w}(\eta)+\mathrm{O}\left(g^{6}\right)
$$

with Wilson fermions and

$$
\tilde{g}_{\eta}^{2} \stackrel{a \rightarrow 0}{=} g_{g w}^{2}+g_{g w}^{4} b_{0} \ln (a / L)+c_{g}^{g w}(\eta)+\mathrm{O}\left(g^{6}\right),
$$

with chiral (gw) fermions. In this formulae, $b_{0}=-N / \pi$, is the correct universal first coefficient of the beta-function (showing asymptotic freedom), which was computed earlier in the continuum [12]. The finite parts found in the two computations differ as expected. The result for the renormalised couplings $\tilde{g}_{V}^{2}$, corresponding to the vector-vector interaction, can be found in ref. [7].

An universal renormalisation group invariant (RGI) quantity can now be defined as the difference of the renormalised coupling for two different values of $\eta$

$$
F_{\mathrm{RGI}}(\eta, a / L)=\tilde{g}_{\eta}^{2}(a / L)-\tilde{g}_{\eta_{0}}^{2}(a / L), \quad \eta_{0}=1 .
$$

This quantity has an expansion in the bare couplings

$$
F_{\mathrm{RGI}}(\eta, a / L)=F_{1}^{(1)}(\eta, a / L) g^{4}+F_{2}^{(1)}(\eta, a / L) g^{2} g_{V}^{2}+F_{3}^{(1)}(\eta, a / L) g_{V}^{4}+\mathrm{O}\left(g^{6}\right),
$$

where the coefficients are given by the finite parts. Since it is an universal quantity, these coefficients have a finite and unique continuum limit independent of the discretisation. The continuum 


\begin{tabular}{|l|l|l|l|l|l|}
\hline$\eta$ & 0.1 & 0.2 & 0.3 & 0.4 & 0.5 \\
\hline$F_{1, \mathrm{w}}^{(1)}(\eta, 0)$ & $5.0797(6)$ & $4.4704(5)$ & $3.6395(3)$ & $2.7629(1)$ & $1.9672(1)$ \\
\hline$F_{1, \mathrm{gw}}^{(1)}(\eta, 0)$ & $5.078(2)$ & $4.469(2)$ & $3.638(2)$ & $2.762(2)$ & $1.966(2)$ \\
\hline
\end{tabular}

Table 1: First order coefficients in the expansion of $F_{\text {RGI }}$ with Wilson (w) and chiral (gw) fermions.

values for different choices of $\eta$ are given in tab. 1. The errors are estimated by fitting the first few terms of the Symanzik expansion of lattice diagrams to the data at lattice sizes from $4 \times 8$ to $64 \times 128(128 \times 256)$ for chiral (Wilson) fermions (method explained in appendix D of [13]). The continuum values agree within the error, thus confirming universality for the operator (1.3).

\section{Final remarks}

The formulation of the SF for fermionic models of the Gross-Neveu type in [7] has enabled the first test of the recently proposed chiral Dirac operator in the presence of the SF boundary conditions. At 1-loop we have shown that a chiral Ward identity takes its continuum value far away from the boundaries. The calculation of an universal quantity gives the right discretisation independent value.

The size of the lattice artifacts has not been discussed so far. What we observe is that the chiral fermions are tree-level $\mathrm{O}(a)$-improved, if the value of $c$ is tuned correctly. At 1-loop it is not enough to tune $c$, because there are dimension 3 operators at the boundary, that spoil automatic $\mathrm{O}(a)$-improvement. This however is peculiar to the studied kind of models with four-fermion interactions.

As next step, it would be desirable to perform a similar (perturbative) computation in QCD in order to provide guidance for the final non-perturbative application of chiral fermions in the Schrödinger functional.

Acknowledgement We would like to thank Rainer Sommer critical reading of the text.

\section{References}

[1] M. Luscher, JHEP 0605 (2006) 042 [arXiv:hep-lat/0603029].

[2] D. J. Gross and A. Neveu, Phys. Rev. D 10 (1974) 3235.

[3] R. Sommer, Nucl. Phys. Proc. Suppl. 160 (2006) 27 [arXiv:hep-ph/0607088].

[4] S. Sint, Nucl. Phys. B 421 (1994) 135, Nucl. Phys. B 451 (1995) 416

[5] S. Sint, PoS LAT2005 (2006) 235 [arXiv:hep-lat/0511034].

[6] P. H. Ginsparg and K. G. Wilson, Phys. Rev. D 25 (1982) 2649.

[7] B. Leder, PhD Thesis, arXiv:0707.1939 [hep-lat].

[8] M. Luscher, S. Sint, R. Sommer and P. Weisz, Nucl. Phys. B 478 (1996) 365 [arXiv:hep-lat/9605038].

[9] P. Hernandez, K. Jansen and M. Luscher, Nucl. Phys. B 552, 363 (1999) [arXiv:hep-lat/9808010].

[10] L. Giusti et al., Comput. Phys. Commun. 153 (2003) 31 [arXiv:hep-lat/0212012].

[11] M. Luscher, Phys. Lett. B 428 (1998) 342 [arXiv:hep-lat/9802011].

[12] P. K. Mitter and P. H. Weisz, Phys. Rev. D 8 (1973) 4410.

[13] A. Bode, P. Weisz and U. Wolff [ALPHA collaboration], Nucl. Phys. B 576 (2000) 517 